Engagement work with local health providers/community groups guided development of service provision. The service was promoted through local media and social media sources. Volunteer recruitment, training and ongoing supervision were established. Interventions offered are monthly bereavement support/weekly counselling sessions depending on assessment of risk and need. Sessions delivered via telephone/digital platform. Referrals accepted from health care professionals as well as self-referral pathways.

Results Between September 2020 and June 2021, 34 referrals were received with 199 contacts. Patients on average had six contacts from a counsellor or highly trained volunteer. The volunteer workforce makes up $80 \%$ of team and are invaluable to continued service. Qualitative patient feedback has been positive.

'For me it was perfect, 100\% what I needed. Being able to talk and offload my feelings has helped so much... having someone listen to me and tell me that all I was feeling was normal.'

Conclusions Marie Curie Bereavement services need to adapt to meet the changing needs and demands in response to pandemic. Engagement work across the community has been integral to the development, outreach and promotion of the service.

\section{THINKING DIFFERENTLY AND THINKING QUICK IN THE MIDDLE OF A PANDEMIC}

Megan Boyle, Aneta Saunders, Kate Heaps. Greenwich and Bexley Community Hospice, London, UK

\subsection{6/spcare-2021-Hospice.118}

Background The effects of the COVID-19 pandemic dramatically diminished the income generation capacity of hospices and posed significant risk to their ability to meet increasing need. According to one survey, 29\% of charities were not confident they would be able to continue post-lockdown (Blackbaud \& Chartered Institute of Fundraising, 2020). The importance of the decisions made in response cannot be overlooked. While no one can offer the 'right answer', even with hindsight, our hospice embraced an agile, go-getting approach that reaped reward and set us up for a promising year.

Aims Our priority was to protect the financial position of the hospice, maximising on opportunities to generate income.

Methods We chose not to furlough our fundraisers. We applied an agile decision making process and utilised our resources flexibly, re-allocating team time. This included whole-team support for the emergency appeal, covering hospice reception duties, securing PPE and supporting clinical teams with testing. We embraced digital channels and delivered an emergency appeal within weeks of lockdown. Our team, empowered to work differently, delivered an almost complete calendar including virtual mass participation challenges and a Christmas grotto.

Results

- Emergency appeal raised $£ 400 \mathrm{~K}+$.

- Overall income exceeded budget.

- 195 hours supporting the inpatient unit with testing.

- Working closer with colleagues has improved perception of the fundraising team and fostered a mutual respect across the organisation.

- Fundraisers remained connected with the cause.
- Thinking creatively, remaining agile and contingency planning have become habit.

- Trustees have better understanding of value and reach of our teams' work.

- We laid foundations for further development of key income streams.

Conclusion Investing in the right way and at the right time was a better strategy for us than to focus on cost management. An ambitious, bold approach powered new ways of working which proved fruitful and has shaped team practice and culture going forward.

\section{P-102 EMPOWERING FUNDRAISERS TO RESPOND TO THE FUNDRAISING GAP CAUSED BY THE PANDEMIC}

Ann-Marie Kelly, Cheri Strudwick. Heart of Kent Hospice, Maidstone, UK

10.1136/spcare-2021-Hospice. 119

When restrictions were enforced in March 2020, Heart of Kent Hospice feared the writing was on the wall - we faced very real risk of closure. Although we had grown our reserves, we had three months to find a solution and fill a projected $£ 800,000$ gap in funding generated through the closure of shops and cancelled fundraising events. We aimed to:

- Be transparent about the challenges we were facing with colleagues.

- Be honest about the scale of the challenge with our supporters.

- Retain donor relationships and recruit additional support.

- Look after the emotional wellbeing of our colleagues as they transitioned to home working.

- Fill the financial gap that had been created to secure the future of the hospice.

Clear guidance was provided on the potential scale of the challenge to the fundraising and communications team and we re-forecast organisational expenditure assuming reaching a worst case scenario and meeting the needs of an increasing caseload of patients.

The fundraising and communications team were trusted to be creative and:

- Respond to the eagerness of the local community to want to help us.

- Try new ideas for income generation - we had nothing to lose!

- Seek alternative ways to engage with our local community who were experiencing sudden loss; we launched a schools bereavement project and guidance for companies.

- Look after each other.

Our urgent fundraising appeal raised $£ 1.2$ million including a grant of $£ 500,000$ to support our recovery post pandemic. Our worst and best case fundraising targets were exceeded.

As a consequence, we have invested in fundraising resource this year to encourage donors to continue supporting us during the rest of the pandemic and into recovery. Heart of Kent Hospice did not furlough any of the fundraising and communications team; instead, we used agility and creativity to survive and strengthen our potential to recover fundraising. 